\title{
Analysis of Operation Room in Laparoscopic Colorectal Cancer Radical Operation
}

\author{
Chen Jie* \\ Operation Room, Renhe Hospital of Beijing, Beijing 102600, China
}

\begin{abstract}
Objective: To analyze the effect of operation room combined with laparoscopic colorectal cancer radical resection, for clinical reference. Method: From July 2011 to June 2012 in our hospital, 62 cases of laparoscopic colorectal cancer radical resection as the observation group, which treated with optimize operation room nursing. In the same period, 58 patients were treated with routine nursing, and the clinical data were retrospectively analyzed, and the difference of post-operative complications and nursing satisfaction rate of the two groups were compared. Results: Compared with the control group, the incidence of post-operative complications was significantly lower in the observation group, and nursing quality satisfaction rate was higher, there was significant difference between the two groups $(p<0.05)$. Conclusion: Quality of operation room nursing care reduces complications of laparoscopic colorectal cancer resection, improve quality of care, reduce patient's pain, and it is worth to be promoted in operation room nursing work in the future.
\end{abstract}

\author{
Keywords \\ Operation room \\ Laparoscopic \\ Colorectal cancer \\ Radical operation
}

\section{Introduction}

In recent years, the incidence of colorectal cancer has gradually increased, which brings serious threat to human life. In the treatment of colorectal cancer, laparoscopic colorectal cancer surgery is more common, and this kind of surgery has the advantages of less trauma, less pain and good tolerance, etc. To a certain extent, it improve the patients' quality of life. Colorectal cancer is a common malignant tumor of the gastrointestinal tract, and the current clinical treatment is still based on surgical resection. With the development of laparoscopic surgery, laparoscopic colorectal cancer radical operation with its advantages of less trauma, less bleeding, faster recovery, etc., hence it more widely used in clinical [1]. The author analyzes the effect of operation room combined with laparoscopic colorectal cancer radical operation, and the results are as follows:

Copyright $\odot 2012$ Chen Jie

doi: 10.18686/aem.v1i1.2

Received: April 16, 2012; Accepted: June 20, 2012; Published online: August 19, 2012

This is an open-access article distributed under the terms of the Creative Commons Attribution Unported License (http://creativecommons.org/ licenses/by-nc/4.0/), which permits unrestricted use, distribution, and reproduction in any medium, provided the original work is properly cited.

${ }^{\star}$ Corresponding author: Operation Room, Renhe Hospital of Beijing, Beijing 102600, China. E-mail: drchen_j103@163.com

\section{Materials and methods \\ 2.1. General information}

62 patients hospitalize during July 2011 to June 2012 in our hospital with laparoscopic radical resection of colorectal carcinoma as the observation group, wherein 40 cases of men and 22 cases of women; aged from 40 to 72 years old, and average age was $(63.14 \pm 9.36)$ years. Their weight range from $55-78 \mathrm{~kg}$ and average weight was $61.44 \mathrm{~kg}$. From there, 12 cases of ascending colon cancer, 4 cases of colon cancer, 15 cases of sigmoid colon cancer, 31 cases of rectal cancer; and by Dukes classification, A stage were 5 cases, 33 cases were in stage $B$, and stage $C$ were 24 cases.

58 patients with rectal cancer who underwent laparoscopic radical resection in our hospital from June 2011 to July 2010 which 20 male and 38 female, aged from 42 to 75 years, average age was $(62.85 \pm 9.14)$ years old, weight $53 \mathrm{~kg}$, average weight was $(61.25 \pm 7.38)$ Dukes; 10 cases of ascending colon cancer, 5 cases of descending colon cancer, 13 cases of sigmoid colon cancer, 30 cases of rectal cancer, 4 cases of A stage, 32 cases of B stage and 22 cases of C stage.

All patients were diagnosed by colonoscopy and pathology. Compared with the two groups of patients with general information, the gender, age, weight, tumor type, clinical stage and so on were not statistically significant difference $(p>0.05)$, with good comparability. 


\subsection{Intervention method}

In the control group, the patients in the perioperative period were given routine nursing intervention, and the observation group was optimized with the operation room.

On the first day of the visit, patients were treated with advanced nature and safety of laparoscopic operation in order to relieve the patients. Explain the possible cases and the key points in operation to guide the patients to prepare mentally and physically [2].

The equipment nurse and circuit nurse pre-operative examination of the surgical instruments, gauze, etc., and detailed record. It is important to familiar with all kinds of instrument connection mode and operation note. Adjust the instrument parameters according to the operation requirements, connect the pipe lines, and adjust the peritoneal lens clarity. Before insert the lens into the abdominal cavity, it should wipe with Iodophor gauze to prevent cold mirror surface condensation generated after entering the peritoneal cavity and affect the vision of surgeon.

Patients that send into the operation room should check carefully the information and chat with patients to ease the tension. Establishment of upper extremity venous channels effectively, assist the anesthesiologist for anesthetic procedure and help patients to display appropriate posture. After the success of anesthesia, patients' legs will fixed and supporting by leg rack to maintain proper tightness. Besides it should cover with leg pad cushion in order to avoid oppression caused injury of common peroneal nerve. The establishment of $\mathrm{CO}_{2}$ pneumoperitoneum should pay attention to adjust the gas flow, initial with low flow and later with high flow. Closely monitor patient vital signs and blood oxygen saturation index, at the same time, observe the patient's reaction, and when there is any abnormal, report timely to the anesthesiologist and surgeon for processing [3].

After tumor resection, immediately cover the site with aseptic bag for incision protection. This is to prevent cancer cell spread and contaminate, and keep the specimens sent for pathological examination. Help patients recover in supine position after completion of the operation, and pay attention to check the integrity of the skin and keep warm. Instruments and circuit nurse should count the equipment and dressing, while circuit nurse turn off the instrument and cut off the power switch; whereas equipment nurses responsible for equipment cleaning, and routine maintenance.

After completing of surgery, patient was send back to the ward and the transfer of work between circuit nurses and ward nurses.

\subsection{Statistical methods}

All the data were input into SPSS 17.0 software for statistical analysis, count data express as rate (\%), using $x^{2}$ test was used for between group comparison, when $p<0.05$ indicated a statistically significant difference.

\section{Results}

\subsection{Post-operative complications}

Compared with control group, incidence of post-operative complications in observation group was significantly lower, with statistically significant difference $(p<0.05)$. Specific data are shown in Table 1.

\subsection{Comparison of nursing quality satisfaction rate}

Compared with control group, observation group of patients with high quality of care satisfaction, there was a significant difference $(p<0.05)$. Specific data are shown in Table 2.

\section{Discussion}

\subsection{Pre-operative preparation}

3 days before operation, patient were encourage to follow less residue semi liquid diet, 2 days before surgery patient must follow liquid diet and forbidden edible beans, milk and other gas-producing foods; 1 day before surgery, heating $500 \mathrm{~mL}$ of $20 \%$ mannitol mixed $500 \mathrm{~mL}$ of $5 \%$ glucose and normal saline administrated orally, and finish within 1 hour; intestinal antibiotics kanamycin $0.5 \mathrm{~g}+$ metronidazole $0.4 \mathrm{~g}$, was given orally every 2 hours, 4 times in total. During afternoon of pre-operative day and morning or surgery day, the cleansing enema was given to patients [4].

\subsection{Pre-operative visit of the patients}

One day before the surgery, operation nurses should visit

Table 1. Comparison of post-operative complications in two groups (case \%).

\begin{tabular}{ccccc}
\hline Group & Anastomotic leakage & Infected & Bleeding & Complication rate \\
\hline Control group $(\mathrm{n}=58)$ & $2(3.45)$ & $4(6.90)$ & $3(5.17)$ & $9(15.52)$ \\
Observation group $(\mathrm{n}=62)$ & $0(0.00)$ & $2(3.23)$ & $1(1.61)$ & $3(4.84)^{*}$ \\
\hline
\end{tabular}

Note: Compared with the control group, ${ }^{*} p<0.05$.

Table 2. Comparison of nursing quality satisfaction rate in two groups (case \%).

\begin{tabular}{ccccc}
\hline Group & Very satisfied & Comparatively satisfied & Dissatisfied & Satisfaction rate \\
\hline Control group $(n=58)$ & $34(58.62)$ & $20(34.48)$ & $4(6.90)$ & $54(93.10)$ \\
Observation group $(n=62)$ & $45(72.58)$ & $17(27.42)$ & $0(0.00)$ & $62(100.00)^{*}$ \\
\hline
\end{tabular}

Note: Compared with the control group, ${ }^{*} p<0.05$. 
the patient voluntary to introduce the environment of the surgery room, the procedure and etc. Besides that, inform the patients regarding the advantages of laparoscopic surgery to reduce the mental stress of patient, so that they can face the operation with a better attitude.

\subsection{Equipment preparation}

Pre-operative preparation of the instruments should do before surgery. Prepare a set of laparoscopic instruments, $45-50{ }^{\circ} \mathrm{C}$ of sterile water, hemostatic gauze, specimen bags, etc. Operation room nurses must be familiar with the performance of instrument, equipment, and proper use of equipment, cleaning, sterilization and maintenance methods.

\subsection{Cooperation of equipment nurses}

Equipment nurses were required to enter the stage 20 min before the surgery to check and arrange the material and equipment at the same time ensure all the screws was tightly locked. The sterilized instruments are placed accordingly on the table of the operation, and all the equipment was adjusted to the best state. Followed by disinfection and then spread towels, together with the circuit nurses carefully counting equipment, yarn, all kinds of wires, and various wiring and piping should properly fixed in the sterile bed. During the operation, instrument nurse need to highly focused and actively cooperate with the work, by fast and accurate delivery of a variety of equipment, and at any time wipe the lens with iodophor gauze, to prevent lens atomization and affect the operation.

\subsection{Cooperation with circuit nurses}

Carefully checks the wristband and data of patients, and help patients adjust to the correct posture. At the same time, pay attention to the patient's respiratory and circulatory function, fully exposed to the surgery field. Besides, establishment of peripheral venous access, assist the anesthesiologist for tracheal intubation, secure fixation of patients. The changes of vital signs such as blood pressure, blood pressure, arterial blood pressure, urine volume and so on, according to the monitoring results, the transfusion rate and fluid volume were adjusted according to the monitoring results. With the assist of equipment nurses together carefully check and count for the inventory items, and the results recorded in the nursing surgical record. Escorting patients back after patient awake from the surgery with the assist of anesthesiologist.

With the deterioration of the environment and changes of diet structure, incidence of colorectal cancer is increasing year by year. Laparoscopic colorectal cancer radical operation has the advantages of minimally invasive and wound as well as quick recovery, but the operation is difficult, time interval was long, requiring surgery doctor with a wealth of experience and skills in laparoscopic operation, the requirements for the cooperation of the operation room nursing care is also higher. Operation room nurses must fully understand the operation procedures of laparoscopic colorectal cancer radical operation, surgical site of the anatomical structure, accurate, effective with the operation, hence reduce the operation accident and risk to a minimum.

Laparoscopic radical resection of rectal carcinoma in the treatment of colorectal cancer clinical effect is more significant, to a certain extent, improve the living standards of patients. All kinds of negative emotions can be easily occurred in the diagnosis of colorectal cancer patients, which brings serious threat to the life of patients, and has certain effect on the clinical treatment effect. At this time, the nursing staff should use the corresponding nursing measures to carry out nursing management to reduce the bad emotional of patients. Therefore, before patients carry out surgery treatment, nursing staff carry out corresponding nursing measures according to the patient's physiological and psychological situation, besides introduce the safety and advantage of the operation to stabilize their emotions, and promote their active cooperation with medical workers [5]. Therefore, the use of laparoscopic colorectal cancer surgery for rectal cancer patients cooperate with the treatment process of operation room nursing achieve a good results, and to a certain extent, it reduce the incidence of complications, and promote the early recovery of patients.

This study found that: the quality of operation room nursing care can reduce the complications of laparoscopic colorectal cancer radical operation, at the same time improve the quality of care and reduce the patient's discomfort. Therefore it is worth to be promoted in the operation room nursing work in the future.

\section{References}

1. Wang JH, Deng FP, Zhang J. The elderly laparoscopic colorectal cancer radical mastectomy perioperative treatment. Clinical and Experimental Medicine. 2012;11(2):130-131.

2. Yang LH, Lv WH, Eileen C. The operation of laparoscopic colorectal cancer resection combined. Shanxi Medical College Journal. 2011;21(1):79-78.

3. Cheng J, Lu Q, Zhao WP. The operation cooperation and nursing experience of laparoscopic colorectal cancer radical resection of rectal cancer. Journal of General Hospital of the Air Force PLA. 2009;36(1):36-37.

4. Mo $\mathrm{CH} .13$ cases laparoscopic colorectal cancer radical surgery operative cooperation and nursing. Modern Chinese Doctor. 2010;48(15):53,70.

5. Liu LH, Xu QP. 28 cases of laparoscopic colorectal cancer radical operation. Chinese Modern Pharmaceutical Applications. 2009;3(3):52-53. 